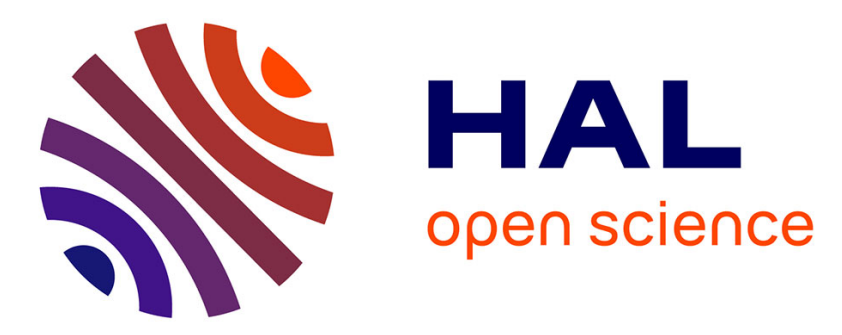

\title{
Broadband photonic transport between waveguides by adiabatic elimination
}

Hassan Oukraou, Virginie Coda, Andon A Rangelov, Germano Montemezzani

\section{To cite this version:}

Hassan Oukraou, Virginie Coda, Andon A Rangelov, Germano Montemezzani. Broadband photonic transport between waveguides by adiabatic elimination. Physical Review A : Atomic, molecular, and optical physics [1990-2015], 2018, 97 (2), pp.023811. 10.1103/PhysRevA.97.023811 . hal-01712962

\section{HAL Id: hal-01712962 \\ https://hal.science/hal-01712962}

Submitted on 16 Apr 2020

HAL is a multi-disciplinary open access archive for the deposit and dissemination of scientific research documents, whether they are published or not. The documents may come from teaching and research institutions in France or abroad, or from public or private research centers.
L'archive ouverte pluridisciplinaire HAL, est destinée au dépôt et à la diffusion de documents scientifiques de niveau recherche, publiés ou non, émanant des établissements d'enseignement et de recherche français ou étrangers, des laboratoires publics ou privés. 


\title{
Broadband photonic transport between waveguides by adiabatic elimination
}

\author{
Hassan Oukraou,,${ }^{1,2, *}$ Virginie Coda, ${ }^{1,2}$ Andon A. Rangelov, ${ }^{3}$ and Germano Montemezzani ${ }^{1,2}$ \\ ${ }^{1}$ Université de Lorraine, LMOPS, 2, rue Edouard Belin, 57070 Metz, France \\ ${ }^{2}$ CentraleSupélec, LMOPS, 2, rue Edouard Belin, 57070 Metz, France \\ ${ }^{3}$ Department of Physics, Sofia University, James Bourchier 5 Boulevard, 1164 Sofia, Bulgaria
}

(Received 6 November 2017; revised manuscript received 22 December 2017; published 6 February 2018)

\begin{abstract}
We propose an adiabatic method for the robust transfer of light between the two outer waveguides in a three-waveguide directional coupler. Unlike the established technique inherited from stimulated Raman adiabatic passage (STIRAP), the method proposed here is symmetric with respect to an exchange of the left and right waveguides in the structure and permits the transfer in both directions. The technique uses the adiabatic elimination of the middle waveguide together with level crossing and adiabatic passage in an effective two-state system involving only the external waveguides. It requires a strong detuning between the outer and the middle waveguide and does not rely on the adiabatic transfer state (dark state) underlying the STIRAP process. The suggested technique is generalized to an array of $N$ waveguides and verified by numerical beam propagation calculations.
\end{abstract}

DOI: 10.1103/PhysRevA.97.023811

\section{INTRODUCTION}

Among the many useful analogies existing between quantum mechanics and classical optics [1-6], the one relating the quantum process of stimulated Raman adiabatic passage (STIRAP) [7-10] and systems of coupled optical waveguides has been widely investigated and applied in recent years [11-18]. Examples includes analogies of STIRAP in a threewaveguide directional coupler [11-13], fractional STIRAP [14], and extensions of STIRAP to multiple states [15-18]. Shortcuts to optical adiabatic passage were also proposed, e.g., in multimode waveguide devices in order to reduce the required length for mode conversion with respect to conventional STIRAP-like schemes [19,20]. An important feature of the above adiabatic light transfer processes is their robustness with respect to the waveguide design parameters, which is related to the fact that the light waves are associated with the same spatially evolving eigenstate of the system. This robustness leads, for instance, to an increased spectral bandwidth.

In the three-waveguide case of STIRAP, the light is transferred adiabatically from an input waveguide 1 to a target waveguide 3 , which are coupled via an intermediate waveguide 2 by two sequential evanescent coupling processes, named pump and Stokes in analogy to the corresponding coupling pulses in atomic physics. A unique and very useful feature of STIRAP is that for a counterintuitive order of couplings, meaning that the Stokes coupling between waveguides 2 and 3 is preceding the pump coupling, the intermediate waveguide 2 does not get any light, even transiently. Therefore, the properties of the intermediate waveguide, including possible losses, are largely irrelevant. The reason for this unique feature is that the evolution of STIRAP proceeds via an adiabatic dark state, which does not involve waveguide 2 . The dark state is associated with waveguide 1 initially and waveguide 3 in the

\footnotetext{
*hassan.oukraou@centralesupelec.fr
}

end, thereby providing a direct adiabatic route from 1 to 3 . It is important to note that this conventional STIRAP works well only for the counterintuitive order of couplings because the dark adiabatic state cannot be used for the intuitive order. This means that the symmetry for left to right is broken.

In this paper, we suggest robust waveguide structures inspired by adiabatic elimination in quantum physics [21-25] that lead to full light transfer independently of the intuitive or counterintuitive order of couplings. Importantly, the proposed technique conserves the symmetry between the left and right waveguides. It involves a detuning between the propagation constants of the outer waveguides and the central waveguide(s) that is constant over propagation. Moreover, in contrast to multiple STIRAP, which can be used successfully to transfer light between the outer waveguides only if the total number of waveguides in the array is odd [15-18], the present approach works equally well for odd and even numbers of waveguides in the array. While most earlier studies on adiabatic elimination in waveguide optics considered only parallel waveguides [26-28], here we take advantage of the simple control parameter of a varying coupling strength to the outer waveguides, which can be realized by varying the distance between these outer waveguides and the central ones [29]. It is shown that these spatially varying coupling constants lead to a spacedependent pseudodetuning of the propagation constants in an effective two-waveguide system. For our specific waveguide design this effective system is fully analogous to the rapid adiabatic passage process (RAP) in two-level quantum systems [9]. Therefore, the behavior of our proposed structures is formally equivalent to the one of a two-waveguide structure with an explicit longitudinal variation and zero crossing of the waveguide detuning parameter. Such a system implements the optical analog to the RAP process and was studied experimentally in a recent work [30]. Section II describes the theory underlying adiabatic elimination in a system of three waveguides, while Sec. III describes how the effect is generalized to a total number $N$ of waveguides. Finally, in Sec. IV, we numerically verify 
(a)

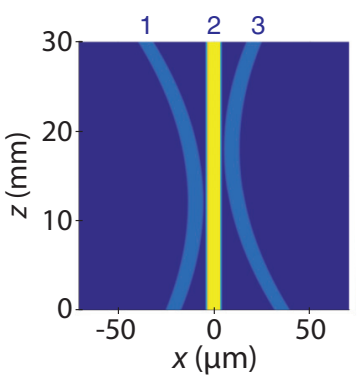

(b)

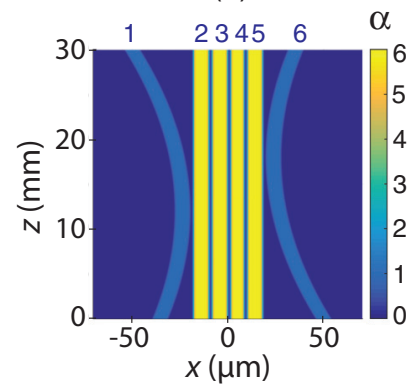

FIG. 1. Waveguide structure for adiabatic elimination in an array of (a) three and (b) six waveguides. The color code visualizes the normalized refractive index contrast $\alpha . \alpha=1$ is the maximum refractive index change of the outer waveguides and $\alpha=6$ the one for the central one(s), leading to a mismatch $\Delta \beta$ of the propagation constants. Injection into the left waveguide 1 at $z=0$ corresponds to an intuitive order of coupling while injection into the right waveguide (waveguide 3, respectively 6) corresponds to a counterintuitive order of coupling.

the expectations by means of the beam propagation method (BPM) and the coupled-mode theory. We analyze the expected spectral and length dependences. The example calculations are performed for fused silica waveguides with propagation around the telecommunication wavelengths and for structures composed of three or six waveguides. It is shown that the combination of adiabatic elimination and of the slow adiabatic spatial evolution of the Stokes and pump coupling constants leads to a robust bidirectional light exchange between the outer waveguides over a broad spectral range.

\section{ADIABATIC ELIMINATION FOR ARRAY OF THREE WAVEGUIDES}

We consider a system of three evanescently coupled dielectric waveguides, such as the one shown in Fig. 1(a), for which the outer waveguides 1 and 3 have an identical refractive index profile which differs from the one of the central one. Let the light propagation constant in the outer waveguides be $\beta_{0}$ and the one of the central waveguide be $\beta_{0}+\Delta \beta$. In the framework of coupled-wave theory, the propagation of the electric field amplitudes $a_{1}(z), a_{2}(z)$, and $a_{3}(z)$ of the waves traveling in the above three waveguides is described by a system of three coupled differential equations written in matrix form as [31]

$$
i \frac{d}{d z}\left[\begin{array}{l}
a_{1} \\
a_{2} \\
a_{3}
\end{array}\right]=\left[\begin{array}{ccc}
0 & C_{12} e^{-i \Delta \beta z} & 0 \\
C_{21} e^{i \Delta \beta z} & 0 & C_{23} e^{i \Delta \beta z} \\
0 & C_{32} e^{-i \Delta \beta z} & 0
\end{array}\right]\left[\begin{array}{l}
a_{1} \\
a_{2} \\
a_{3}
\end{array}\right],
$$

where the $C_{i j}$ are the $z$-dependent coupling coefficients from waveguide $j$ to waveguide $i$ and in general $C_{i j} \neq C_{j i}$. The longitudinal $z$ variation of the coupling constants is associated with the changing distance between the waveguides seen in Fig. 1(a). The direct coupling between waveguides 1 and 3 is neglected because the structure is assumed to be planar and their distance is supposed to be sufficiently large.

Similarly to the approach given in Ref. [32], the above Eq. (1) can be brought in a more symmetric form by

performing the simple transformation $a_{1}^{\prime}=\sqrt{C_{21} / C_{12}} a_{1}$, $a_{2}^{\prime}=\exp (-i \Delta \beta z) a_{2}, a_{3}^{\prime}=\sqrt{C_{23} / C_{32}} a_{3}$. This leads to the form [11]

$$
i \frac{d}{d z}\left[\begin{array}{l}
a_{1}^{\prime} \\
a_{2}^{\prime} \\
a_{3}^{\prime}
\end{array}\right]=\left[\begin{array}{ccc}
0 & C_{P} & 0 \\
C_{P} & \Delta \beta & C_{S} \\
0 & C_{S} & 0
\end{array}\right]\left[\begin{array}{l}
a_{1}^{\prime} \\
a_{2}^{\prime} \\
a_{3}^{\prime}
\end{array}\right],
$$

where the pump $\left(C_{P}\right)$ and the Stokes $\left(C_{S}\right)$ coupling constants are geometrical averages of the above coefficients $C_{i j}$, i.e., $C_{P} \equiv \sqrt{C_{12} C_{21}}$ and $C_{S} \equiv \sqrt{C_{23} C_{32}}$.

When the propagation constant mismatch $\Delta \beta$ is very large, meaning

$$
\frac{|\Delta \beta|}{\sqrt{C_{P}^{2}+C_{S}^{2}}} \gg 1,
$$

as we shall assume for our case, then the derivative of $a_{2}^{\prime}(z)$ varies rapidly and the average value of $a_{2}^{\prime}(z)$ and $a_{2}(z)$ over many cycles will be zero, thus the average of the derivative vanishes. If no light is initially input in the middle waveguide, this extreme limit leads to a strong suppression of the wave amplitude in this waveguide- this effect is known in quantum physics as adiabatic elimination [21-25]. The easiest mathematical way to perform the adiabatic elimination of the middle waveguide is to set $d a_{2}^{\prime}(z) / d z=0$,

$$
\begin{aligned}
i \frac{d}{d z} a_{2}^{\prime} & =0=C_{P} a_{1}^{\prime}+\Delta \beta a_{2}^{\prime}+C_{S} a_{3}^{\prime} \\
& \Rightarrow a_{2}^{\prime}=-\frac{C_{P}}{\Delta \beta} a_{1^{\prime}}-\frac{C_{S}}{\Delta \beta} a_{3}^{\prime},
\end{aligned}
$$

and to replace the resulting expression for $a_{2}^{\prime}(z)$ in the other two equations of (2). After an unimportant phase shift of the electric field amplitudes such that $\tilde{a}_{i}(z) \equiv$ $\exp \left[-i\left(C_{P}^{2}+C_{S}^{2}\right) z /(2 \Delta \beta)\right] a_{i}^{\prime}(z)$, this leads to an equation characterizing an effective two-state system given by

$$
i \frac{d}{d z}\left[\begin{array}{l}
\tilde{a}_{1} \\
\tilde{a}_{3}
\end{array}\right]=\left[\begin{array}{cc}
-\Delta_{\mathrm{eff}} & C_{\mathrm{eff}} \\
C_{\mathrm{eff}} & \Delta_{\mathrm{eff}}
\end{array}\right]\left[\begin{array}{c}
\tilde{a}_{1} \\
\tilde{a}_{3}
\end{array}\right],
$$

where

$$
\Delta_{\text {eff }}=\frac{C_{P}^{2}-C_{S}^{2}}{2 \Delta \beta}
$$

is an effective detuning and

$$
C_{\text {eff }}=-\frac{C_{P} C_{S}}{\Delta \beta}
$$

is an effective coupling coefficient. We will now write Eq. (5) in the so-called adiabatic basis $[9,10,25]$ (for the two-state atom, this is the basis of the instantaneous eigenstates of the Hamiltonian),

$$
i \frac{d}{d z}\left[\begin{array}{l}
b_{1} \\
b_{3}
\end{array}\right]=\left[\begin{array}{cc}
-\sqrt{C_{\mathrm{eff}}^{2}+\Delta_{\mathrm{eff}}^{2}} & -i \frac{d \vartheta}{d z} \\
i \frac{d \vartheta}{d z} & \sqrt{C_{\mathrm{eff}}^{2}+\Delta_{\mathrm{eff}}^{2}}
\end{array}\right]\left[\begin{array}{l}
b_{1} \\
b_{3}
\end{array}\right],
$$

where

$$
\tan 2 \vartheta=\frac{C_{\text {eff }}}{\Delta_{\text {eff }}} .
$$

The connection between the amplitudes $\tilde{a}_{1}$ and $\tilde{a}_{3}$ and the adiabatic ones $b_{1}$ and $b_{3}$ is given by

$$
\begin{aligned}
& b_{1}=\tilde{a}_{1} \cos \vartheta-\tilde{a}_{3} \sin \vartheta, \\
& b_{3}=\tilde{a}_{1} \sin \vartheta+\tilde{a}_{3} \cos \vartheta .
\end{aligned}
$$


When the evolution of the system is adiabatic, $\left|b_{1}\right|^{2}$ and $\left|b_{3}\right|^{2}$ remain constant $[9,10,25]$. Mathematically, adiabatic evolution means that the nondiagonal terms in Eq. (8) are small compared to the diagonal terms and can be neglected. This restriction amounts to the following adiabatic condition on the process parameters $[9,10,25]$,

$$
\left|\frac{d}{d z} \vartheta\right| \ll\left(C_{\text {eff }}^{2}+\Delta_{\text {eff }}^{2}\right)^{1 / 2}
$$

which, using Eq. (9), takes the explicit form

$$
\frac{2\left(C_{\text {eff }}^{2}+\Delta_{\text {eff }}^{2}\right)^{3 / 2}}{\left|\Delta_{\text {eff }} \frac{d}{d z} C_{\text {eff }}-C_{\text {eff }} \frac{d}{d z} \Delta_{\text {eff }}\right|} \gg 1 .
$$

Hence adiabatic evolution requires a smooth enough $z$ dependence of the effective phase mismatch $\Delta_{\text {eff }}$ and of the effective coupling coefficient $C_{\text {eff }}$, a long enough waveguide length, and a large enough coupling. In the adiabatic regime $\left|b_{1,3}\right|^{2}=$ const, but the light intensity contained in waveguides 1 and $3\left(\propto\left|\tilde{a}_{1,3}\right|^{2}\right)$ will vary if the mixing angle $\vartheta$ varies, thus adiabatic evolution can produce energy transfer between the outer waveguides. Note that $C_{\text {eff }}^{2}+\Delta_{\text {eff }}^{2}$ decreases by increasing $\Delta \beta$, therefore a sort of compromise must be found to optimize both criteria (3) and (12).

For the case of our structures shown in Fig. 1(a) and the examples that will be discussed in Sec. IV, the effective phase mismatch $\Delta_{\text {eff }}$ sweeps from some negative to some positive value. In this process the mixing angle $\vartheta$ changes from $\pi / 2$ to 0 . With the light initially in waveguide 1 , the system will stay adiabatically in state $b_{3}$, so that the light will switch to waveguide 3 when $\vartheta$ approaches zero. Therefore the effective phase mismatch sweep will produce complete light switching. This is exactly the mechanism that is at work also in the RAP-like two-waveguide system with explicit detuning that was reported recently [30]. It is worth noting that adiabatic transfer does not bring about any specific restriction to the shape of the effective phase mismatch $\Delta_{\text {eff }}(z)$ as far as the condition (12) is fulfilled and the mixing angle $\vartheta$ changes from $\pi / 2$ to 0 (or vice versa).

The effective phase mismatch $\Delta_{\text {eff }}$ can sweep from negative values to some positive values (or vice versa) if the Stokes coupling is stronger than the pump coupling at the beginning of propagation and the reverse is true at the end of propagation (or vice versa). The consequence is that the intuitive and the counterintuitive order of coupling will lead both to the same final outcome, a complete switching of the light between the outer waveguides in the array, as will be verified in Sec. IV by means of BPM simulations. This property is not found in the case of the conventional STIRAP process.

\section{ADIABATIC ELIMINATION FOR ARRAY OF $N$ WAVEGUIDES}

The above adiabatic elimination effectively keeps only the two outer waveguides and eliminates the effect of the central one. This effect can be extended to the case of an array of $N$ waveguides, where the $N-2$ internal waveguides are adiabatically eliminated, keeping again only the two outer ones. Below, we limit ourselves to the explicit demonstration of the case $N=4$, but the conclusions remain valid in the general case of $N$ states, as was shown in the case of quantum physics [33].

Similar to the case of Eq. (2), the equation describing the evolution of the normalized wave amplitudes $a_{i}^{\prime}(z)$ in an array of four waveguides can be brought in the form

$$
i \frac{d}{d z}\left[\begin{array}{l}
a_{1}^{\prime} \\
a_{2}^{\prime} \\
a_{3}^{\prime} \\
a_{4}^{\prime}
\end{array}\right]=\left[\begin{array}{cccc}
0 & C_{P} & 0 & 0 \\
C_{P} & \Delta \beta & C_{I} & 0 \\
0 & C_{I} & \Delta \beta & C_{S} \\
0 & 0 & C_{S} & 0
\end{array}\right]\left[\begin{array}{l}
a_{1}^{\prime} \\
a_{2}^{\prime} \\
a_{3}^{\prime} \\
a_{4}^{\prime}
\end{array}\right],
$$

where $C_{P} \equiv \sqrt{C_{12} C_{21}}, C_{S} \equiv \sqrt{C_{34} C_{43}}$ are the pump and Stokes couplings, respectively, and $C_{I}=C_{23}=C_{32}$ is the coupling coefficient between the internal waveguides, which are assumed to be identical and are detuned by $\Delta \beta$ with respect to the outer ones. Here, the adiabatic elimination of the intermediate waveguides is done by setting $d a_{2}^{\prime} / d z=0$ and $d a_{3}^{\prime} / d z=0$ in Eq. (13), determining the amplitudes $a_{2}^{\prime}$ and $a_{3}^{\prime}$ in terms of $a_{1}^{\prime}$ and $a_{4}^{\prime}$ from the resulting set of linear algebraic equations, and replacing them in the equations for $d a_{1}^{\prime} / d z$ and $d a_{4}^{\prime} / d z$. This permits one to reduce the four-state problem to an effective two-state system which takes a form identical to Eq. (5),

$$
i \frac{d}{d z}\left[\begin{array}{l}
\tilde{a}_{1} \\
\tilde{a}_{4}
\end{array}\right]=\left[\begin{array}{cc}
-\Delta_{\text {eff }} & C_{\text {eff }} \\
C_{\text {eff }} & \Delta_{\text {eff }}
\end{array}\right]\left[\begin{array}{c}
\tilde{a}_{1} \\
\tilde{a}_{4}
\end{array}\right],
$$

where the relation between the amplitudes $\tilde{a}_{i}$ and $a_{i}^{\prime}$ is again a simple phase shift and

$$
\begin{gathered}
\Delta_{\text {eff }}=\frac{\Delta \beta\left(C_{P}^{2}-C_{S}^{2}\right)}{2\left(\Delta \beta^{2}-C_{I}^{2}\right)}, \\
C_{\text {eff }}=\frac{C_{P} C_{I} C_{S}}{\Delta \beta^{2}-C_{I}^{2}} .
\end{gathered}
$$

The corresponding expressions for $N=6$, a case that will be illustrated below by BPM simulations, are

$$
\begin{gathered}
\Delta_{\text {eff }}=\frac{\Delta \beta\left(\Delta \beta^{2}-2 C_{I}^{2}\right)}{2\left[\left(\Delta \beta^{2}-C_{I}^{2}\right)^{2}-\Delta \beta^{2} C_{I}^{2}\right]}\left(C_{P}^{2}-C_{S}^{2}\right), \\
C_{\text {eff }}=\frac{C_{P} C_{I}^{3} C_{S}}{\left(\Delta \beta^{2}-C_{I}^{2}\right)^{2}-\Delta \beta^{2} C_{I}^{2}} .
\end{gathered}
$$

Since Eqs. (14) and (5) are identical, as was the case for the three-waveguide situation of Sec. II, in the adiabatic regime the present situation is expected to lead to a RAP-like switch of the wave amplitude between waveguides 1 and 4, both for the counterintuitive and intuitive order of couplings.

\section{EXAMPLES}

In this section we verify the above expectations by performing numerical simulations of the wave propagation in structures containing a total of three or six planar waveguides. A BPM method using a split-step Fourier method is used for the simulations $[34,35]$. The corresponding waveguide structures are shown in Figs. 1(a) and 1(b) for $N=3$ and $N=6$, respectively. The waveguides are assumed to be written in fused silica for which the cladding refractive index is 1.4440 at $1550 \mathrm{~nm}$ [36]. In the transverse $x$ dimension, the form of the refractive index profile of each waveguide $i$ is assumed 
to be $\Delta n_{i}(x)=\alpha_{i} \Delta n_{0} \exp \left[-(x / d)^{8}\right]$, where $\Delta n_{i}(x)$ is the amplitude of the refractive index contrast for waveguide $i$ and $\Delta n_{0}=0.004$. For the sake of simplicity, we consider that all waveguides have the same $1 / e$ half width $d=4 \mu \mathrm{m}$ but the internal waveguides have a larger refractive index contrast (by a factor $\alpha=6$ ) with respect to the outer ones (for which $\alpha=1$ ). This produces the mismatch $\Delta \beta$ in the mode propagation constants. While waveguides 2 to $N-1$ are straight, we assume that the two outer waveguides have a parabolic geometrical form given by

$$
\begin{gathered}
\delta x_{1}(z)=-72(z / L-1 / 2+1 / 10)^{2}, \\
\delta x_{N}(z)=72(z / L-1 / 2-1 / 10)^{2} .
\end{gathered}
$$

$\delta x_{1}(z)$ gives the evolution of the center position of waveguide 1 and $\delta x_{N}(z)$ of waveguide $N$ in micrometers. The total propagation length is $L=30 \mathrm{~mm}$. This gives a $z$-longitudinal separation distance between the maximum of the coupling constants $C_{P}$ and $C_{S}$ of $6 \mathrm{~mm}$. In the $x$-transverse dimension, the minimum separation between two adjacent waveguides is $9.6 \mu \mathrm{m}$ (from the center to the center of the waveguides).

First, we consider the case $N=3$ and the situation where $\alpha=1$ for all waveguides, that is, all waveguides have an identical index profile and thus $\Delta \beta=0$. Obviously, in this case adiabatic elimination cannot take place since the condition (3) is not satisfied. Despite that, when the light is injected in the right outer waveguide (counterintuitive order), a complete light transfer to the leftmost waveguide without excitation of the middle one is obtained. This is seen in Fig. 2(a), simulated for a light wavelength of $1550 \mathrm{~nm}$ and corresponding to the standard STIRAP process in a three-waveguide directional coupler discussed earlier [11-13]. In contrast, as can be seen in Fig. 2(b), the reverse situation where the light is injected in the left waveguide (intuitive order) is much more complex and does not lead to a corresponding transfer to the opposite outer waveguide. This is a manifestation of the mentioned asymmetry of the STIRAP process with respect to waveguide exchange.

Figures 2(c) and 2(d) show the situation as the one of Figs. 2(a) and 2(b), but for the case of an increased index contrast for the central waveguide $(\alpha=6)$. In this case the light transfer occurs both from left to right and from right to left in a symmetric way with almost no excitation of the middle waveguide, as expected from the theory in Sec. II. In fact, for the situation of Figs. 2(c) and 2(d), the adiabatic elimination condition (3) is satisfied. As seen in Fig. 3(a), the minimum value of the ratio $|\Delta \beta| /\left(C_{P}^{2}+C_{S}^{2}\right)^{1 / 2}$ is approximately 4 (obtained at the $z$ locations where either $C_{P}$ or $C_{S}$ is maximal). Note that, as discussed in more detail below, the central waveguides are multimodes. This minimum (worstcase) ratio corresponds to the coupling of the fundamental mode of the outer waveguides (mode number $m=0$ ) with the second excited mode of the central waveguide $(m=2)$. The minimum of the above ratio for the coupling between the fundamental modes of all waveguides is roughly 20 times larger [see Fig. 3(a)]. The corresponding evolution of the adiabatic criterion (12) is shown in Fig. 3(d). If we consider the coupling between the modes $m=0$ and $m=2$, the ratio in (12) varies between 1.5 and 4 over the interacting distance (a)

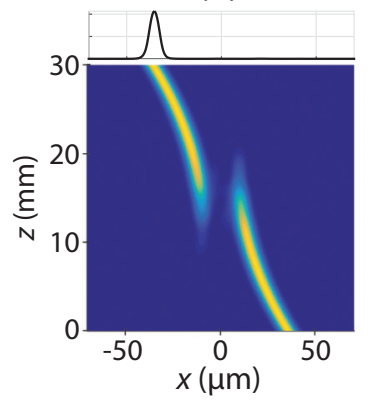

(c)

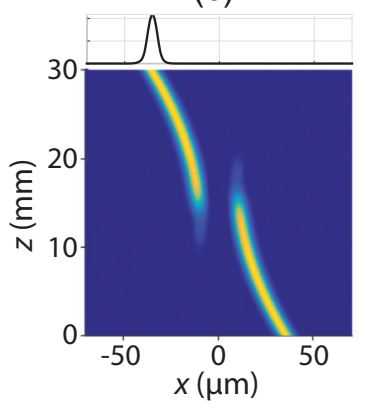

(e)

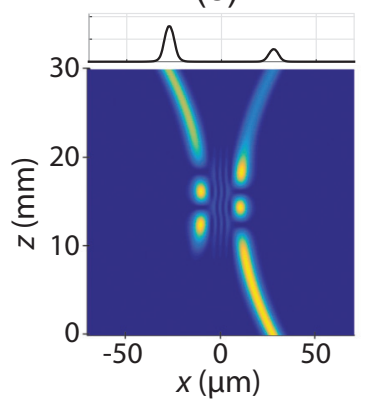

(b)

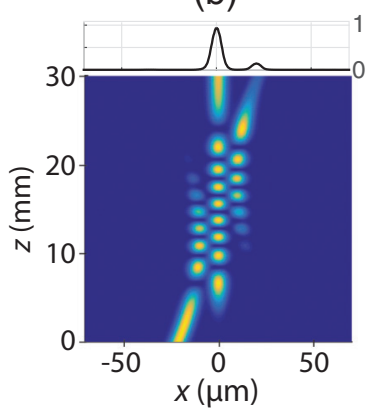

(d)

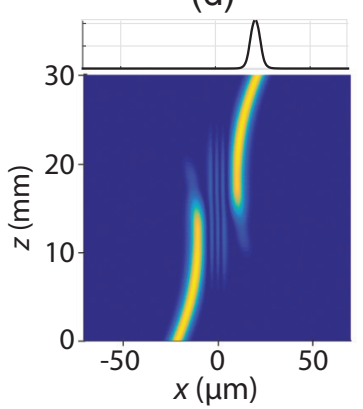

(f)

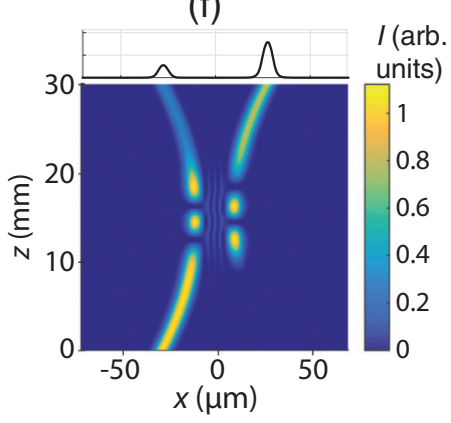

FIG. 2. Wave propagation in a three-waveguide structure calculated by the BPM method at the wavelength $\lambda=1550 \mathrm{~nm}$ for light injection into the right waveguide (left column) and into the left waveguide (right column). (a) and (b) are for the case $\alpha=1$, for which all waveguides have the same index contrast and therefore $\Delta \beta=0$. (c) and (d) are for $\alpha=6$ [refractive index design corresponding to the one of Fig. 1(a)]. This gives the RAP-like light switching between the outer waveguides. (e) and (f) are for a fully symmetric waveguide structure for which $\Delta_{\text {eff }}=0$ in (5) and for which the RAP-like transfer cannot take place $(\alpha=6)$. The small top diagrams represent the intensity profiles at the end of the $30-\mathrm{mm}$ propagation. The intensity color scale is the same for all the surface plots.

where the longitudinal shift between waveguides 1 and 3 leads to a variation of the effective $z$-dependent detuning $\Delta_{\text {eff }}$. In contrast, the ratio of Eq. (12) would be less than one if two fundamental modes are considered since the coupling is weaker and the detuning larger. Therefore, for this coupling among the modes $m=0$, there is an increase of the ratio of Eq. (3) that goes at the expense of the adiabatic criterion (12). Figure 2(d) clearly shows that the mode with $m=2$ appears weakly in the transient regime. Therefore, under the above conditions, the system chooses the channel over the intermediate mode $m=2$ that leads to the optimum transfer and fulfills satisfactorily both criteria. The above values for 
(a)

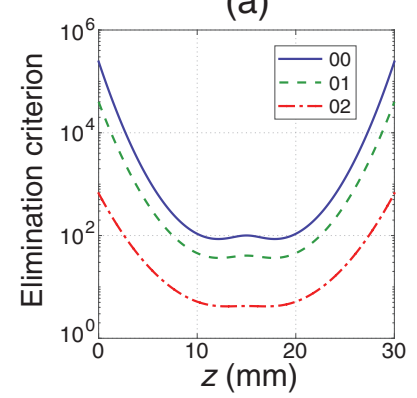

(d)

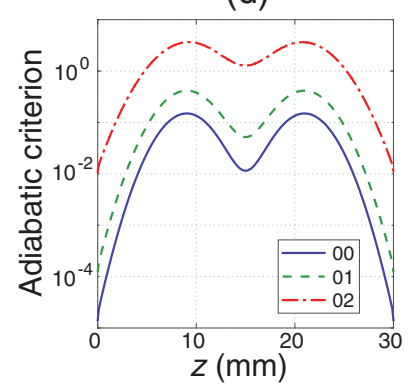

FIG. 3. Visualization of the adiabatic parameters for the threewaveguide case $(N=3)$ for the structure of Fig. 1(a) at $\lambda=1550 \mathrm{~nm}$, corresponding to the symmetric light transfer of Figs. 2(c) and 2(d). The different " $0 i$ " curves consider a possible coupling between the fundamental mode $(m=0)$ of the outer waveguides ( 1 or 3$)$ and the $i$ th mode ( $m=i, i=0,1$, or 2$)$ of the central waveguide 2 . The panels show (a) the $z$ dependence of the adiabatic elimination criterion of Eq. (3), (b) $\Delta_{\text {eff }}(z)$ [Eq. (6)], (c) $\left|C_{\text {eff }}(z)\right|$ [Eq. (7)], (d) the adiabatic criterion of Eq. (12) [deduced from (b) and (c)], and (e) the corresponding RAP-like two-waveguide structure underlying Eq. (5). In (e), the color scale illustrates the variation of the waveguide refractive index contrast with respect to the constant refractive index contrast $\Delta n_{0}=0.004$ of the left waveguide.

criteria (3) and (12) have been approximated by calculating the propagation and coupling constants of the different modes of a planar step index waveguide with a refractive index contrast of $\Delta n_{0}$ and a width equal to the full width at half maximum of the waveguides used in the BPM simulations $(\mathrm{FWHM}=7.64 \mu \mathrm{m})$.

In the case of the symmetric transfer of Figs. 2(c) and 2(d), it is interesting to visualize the evolution of the effective detuning $\Delta_{\text {eff }}(z)$ [Fig. 3(b)] and of the effective coupling $C_{\text {eff }}(z)$ [Fig. 3(c)], as well as the corresponding effective RAP-like waveguide structure associated with Eq. (5), which is shown in Fig. 3(e). Clearly, as seen in Figs. 3(b) and 3(c), only the combination of the fundamental modes in the outer waveguides and the $m=2$ mode in the central waveguide leads to significant values for $\Delta_{\text {eff }}$ and $C_{\text {eff }}$, which confirms again the above reasoning. It is also worth noting that, as seen in Fig. 3(e), the relative index contrast that would be needed to induce the above effective detuning $\Delta_{\text {eff }}$ is quite small. Nevertheless, this is sufficient to induce a robust RAP-like light transfer similar to the cases treated explicitly in Ref. [30].

Obviously, besides adiabatic elimination, also a large enough effective detuning $\Delta_{\text {eff }}$ in (6) is essential for the symmetric transfer observed in Figs. 2(c) and 2(d). To show (a)

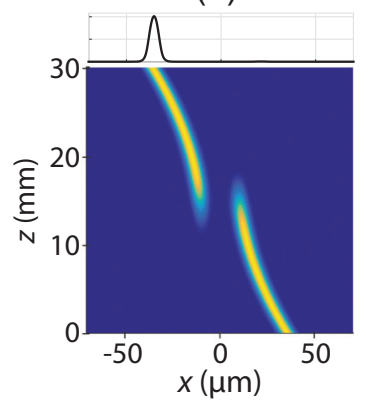

(c)

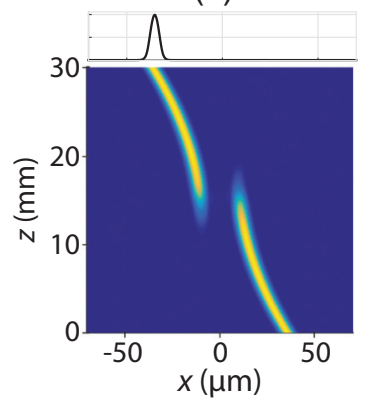

(b)

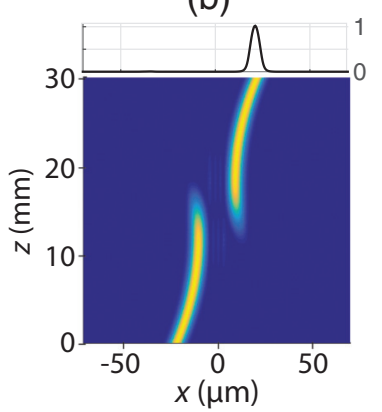

(d)

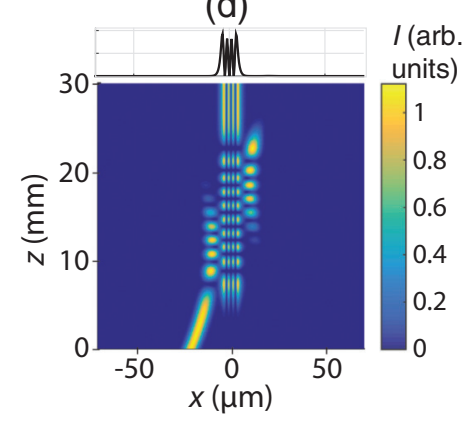

FIG. 4. Wave propagation in a three-waveguide structure as in Figs. 2(c) and 2(d) $(\alpha=6)$ but for different wavelengths. (a) and (b) are at $\lambda=1300 \mathrm{~nm}$, and (c) and (d) are at $\lambda=1137 \mathrm{~nm}$.

this, we also consider as a counterexample the fully symmetric structure where the distances between the two outer waveguides and the central one are identical for all $z$, i.e., $\delta x_{1}(z)=-\delta x_{2}(z)=-72(z / L-1 / 2)^{2}$ instead of Eqs. (19a) and (19b). In this case, the pump and Stokes coupling constants are the same and the pseudodetuning $\Delta_{\text {eff }}$ in Eq. (5) vanishes everywhere. Therefore, the system cannot behave as a RAP process, even though adiabatic elimination of the middle waveguide is still at work. This is seen in Figs. 2(e) and 2(f), which shows how the light is getting distributed among the two outer waveguides.

Figures 4(a) and 4(b) represent the same situations as Figs. 2(c) and 2(d) but for a propagating wavelength of 1300 $\mathrm{nm}$ instead of $1550 \mathrm{~nm}$. A symmetric transfer is also achieved here. The results are even better for this set of parameters, with less transient light in the central waveguide in the intuitive case [Fig. 4(b)]. This is expected since the minimum value of the adiabatic elimination ratio in Eq. (3) is now approximately 22 , again for the coupling of the modes $m=0$ and $m=2$. Note that transfer is efficient despite the fact that the ratio of Eq. (12) is not so large in this case $(\lesssim 1)$. The behavior seen in Figs. 2(c) and 2(d) and Figs. 4(a) and 4(b) proves the robustness of the process resulting in a broadband behavior, which will be discussed in more detail below. This robustness is associated with the adiabatic evolution of the parameters in the RAP-like light transfer between the outer waveguides.

Next, we consider a case where there are more than three waveguides. It is worth remembering that a multistate STIRAP permits one to achieve a robust transfer from the initial to the target state via the so-called adiabatic dark state only if the total number of states is odd and the Stokes coupling precedes the pump coupling (counterintuitive order) [18,33]. 
(a)

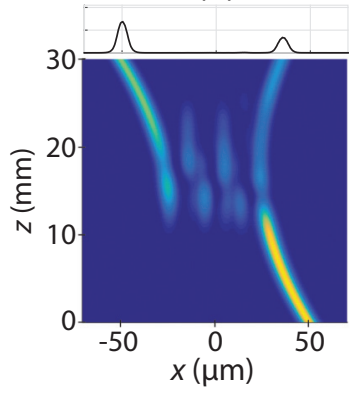

(c)

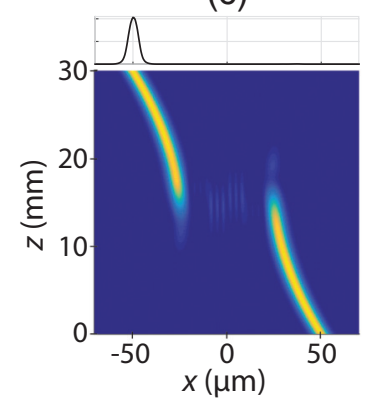

(b)

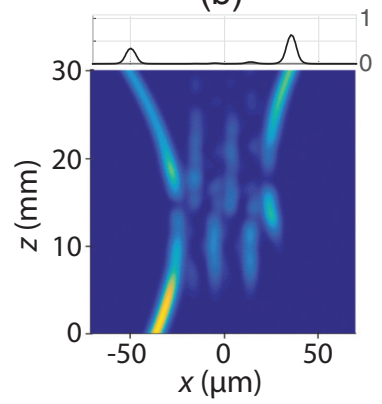

(d)

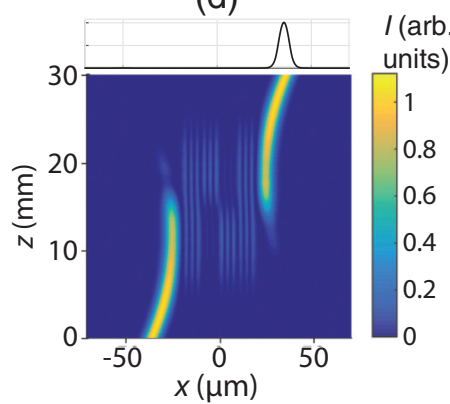

FIG. 5. Wave propagation in a six-waveguide structure calculated by the BPM method for light injection into the right waveguide (left column) and into the left waveguide (right column). The wavelength is $\lambda=1550 \mathrm{~nm}$. (a) and (b) are for the case $\alpha=1$. (c) and (d) are for $\alpha=$ 6 [refractive index design corresponding to the one of Fig. 1(b)]. This gives the RAP-like light switching between the outer waveguides.

For our calculations we choose therefore an even total number of waveguides $(N=6)$ [see Fig. 1(b)] in order to prove that for the present approach there is no limitation on the parity of the waveguide number. Figures 5(a) and 5(b) show again the case where there is no adiabatic elimination because all the waveguides have the same index contrast $(\alpha=1)$ and thus $\Delta \beta=0$. In this case, the propagation behavior is very sensitive to the waveguide geometries and, in general, one obtains a quite complex distribution of the light among the various outputs. In contrast, by increasing the propagation constant mismatch by means of the factor $\alpha=6$, the switch of the wave between the outer waveguides expected from the RAP-like process is observed. This is seen in Figs. 5(c) and 5(d) for $\lambda=1550 \mathrm{~nm}$.

As already mentioned, an important point is that for our simulated cases the outer waveguides are essentially single mode, however, the internal waveguides are no longer single mode as soon as $\alpha>1$. This can be seen, for instance, in Fig. 2(d) where the transient light in the central waveguide for the intuitive case (right column) propagates in the thirdorder mode (with a propagation constant $\beta_{2}$ ). This is the higher supported mode of waveguide 2 , and this mode has therefore the smallest $\Delta \beta$ with the propagation constant $\beta_{0}$ of waveguides 1 and 3 . As a matter of principle, the fundamental mode in the input waveguide can couple to any of the modes of the internal ones. The RAP-like switching behavior can be obtained provided that criterion (3) is satisfied for any of the modes, in other words, all of the propagation constant must be sufficiently far from the propagation constant of the fundamental mode in the outer waveguides. If this is not

(a)
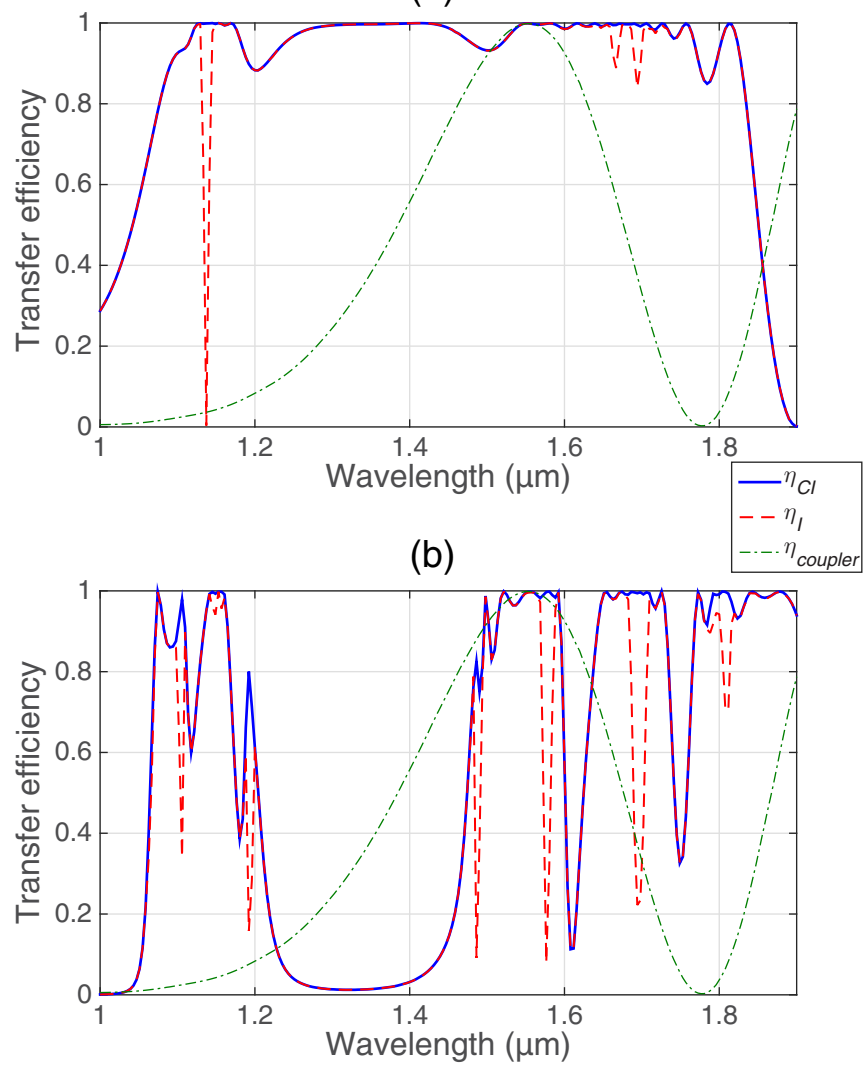

FIG. 6. Evolution of the transfer efficiencies with wavelength for (a) $N=3$ and (b) $N=6$. The refractive index profiles correspond to the ones of Fig. 1. $\eta_{C I}\left(\eta_{I}\right)$ is the transfer efficiency for the counterintuitive case (the intuitive case). The efficiency spectrum is compared to the one of a directional coupler $\left(\eta_{\text {coupler }}\right)$ composed of two straight waveguides and tuned to a wavelength of $1.55 \mu \mathrm{m}$ (see text).

satisfied, the transfer between the outer waveguides is no longer efficient. Therefore, the influence of the competition between modes affects the robustness of the transfer efficiencies. They are defined by $\eta_{C I}=P_{1}(z=L) / P_{N}(z=0)$ for the counterintuitive case and $\eta_{I}=P_{N}(z=L) / P_{1}(z=0)$ for the intuitive case, where $P_{i}(z)$ is the power in waveguide $i$ at the propagation distance $z$. This is illustrated in Fig. 6, representing the transfer efficiencies $\eta_{C I}$ and $\eta_{I}$ for $N=3$ and $N=6$ as a function of wavelength. The dispersion of the refractive index is given by Ref. [36], but we neglect the wavelength variation of $\Delta n_{0}$, $\Delta n_{0}=0.004$, for all wavelengths.

The robustness of the transfer by adiabatic elimination is clearly seen for the three-waveguide case, where a rather flat profile is obtained over more than $600 \mathrm{~nm}$ [Fig. 6(a)]. The efficiency drop for short wavelengths $(\leqslant 1150 \mathrm{~nm})$ is due to a combination of two factors. The first is the decrease of the coupling constants with decreasing wavelengths, and the second is the appearance of the fourth mode in the central waveguide, whose propagation constant $\beta_{3}$ starts close to the fundamental one $\beta_{0}$ of the outer ones, so preventing the adiabatic elimination. For the intuitive case, the efficiency even drops to zero for a specific wavelength around $1.14 \mu \mathrm{m}$ due to the association of an extremely small detuning in 
the propagation constants $\beta_{3}$ and $\beta_{0}$ and a coupling effect driving all the light in the central waveguide at the end of the propagation [see Fig. 4(d)]. For the counterintuitive case, the efficiency $\eta_{C I}$ is still 1, as illustrated in Fig. 4(c), since there is never any light in waveguide 2 in the counterintuitive case (as for STIRAP). Therefore, for specific wavelengths and/or specific values of $\alpha$, the structure might act as a mode converter or a mode filter rather than as a robust directional coupler. For longer wavelengths, the efficiency drops again since the wavelength approaches the cutoff frequency of the third mode and $\beta_{2} \rightarrow \beta_{0}$. This is also due to a too strong coupling. Indeed, for $\lambda>1.8 \mu \mathrm{m}$, for the counterintuitive case (the intuitive case), the light is integrally transferred from waveguide 1 (waveguide 3) to waveguide 3 (waveguide 1) during the propagation, but comes back to waveguide 1 , thus decreasing $\eta$.

The case where all waveguides remain single mode has not been considered since the large required detuning $\Delta \beta$ leads naturally to a multimode structure of the stronger waveguides, at least for realistic simple waveguide profiles as the one considered in this work. Even though the spectral dependence of our proposed coupler is influenced by the multimode behavior and by the wavelength dependence of the coupling, its bandwidth is much larger that the one of a coupler consisting of only two waveguides, as shown with the curve of $\eta_{\text {coupler }}$ in Fig. 6. The latter has been obtained by simulating the wave propagation in two straight coupled waveguides separated by $19.2 \mu \mathrm{m}$ (instead of $9.6 \mu \mathrm{m}$ ). All the others parameters remain the same, especially the refractive index profile $\Delta n(x)$ is the one of waveguides 1 and 3 . The separation distance has been adjusted to get one coupling length for the $30-\mathrm{mm}$ propagation length at $\lambda=1550 \mathrm{~nm}$.

For the six-waveguide cases [Fig. 4(b)], the expected bandwidth is much smaller than for $N=3$. This is related to the fact that, as for the case of a multi-STIRAP process $[18,33]$, the adiabatic condition is more difficult to fulfill with an increased number of waveguides, leading to less robust behavior. The adiabatic criterion (12) for $N=6$ can reach high values $(\gg 1)$ at the $z$ locations of the maximum of $C_{P}$ and $C_{S}$, but drops to $\ll 1$ at $z=L / 2 . \eta$ is therefore much more affected by the wavelength dependence of the coupling constants than for $N=3$. Nevertheless, as for $N=3$, for specific (pseudoperiodic) wavelength ranges, an almost flat transfer efficiency close to 1 is observed also in this case.

All the above examples show that adiabatic elimination can be used to realize a symmetric coupler, but no attempt was made to optimize the dimensions of the related structures. Generally, it is well known that robust adiabatic approaches tend to require longer propagation lengths than nonadiabatic ones. In this context, computational approaches such as those based on shortcuts to adiabacity $[19,20]$ can reduce the device footprint. To judge the possibility of using shorter structures in our specific case, we may consider first the evolution of the effective parameters $\Delta_{\text {eff }}$ and $C_{\text {eff }}$ in Figs. 3(b) and 3(c). One sees that these parameters vary significantly only in a spatial region of the order of $15 \mathrm{~mm}$ around the center of the 30-mm-long structure. One may therefore expect that a certain reduction of the structure length should be possible.

We investigate the tolerance of the coupler for $N=3$ with respect to a variation of the total length $L$ with the help

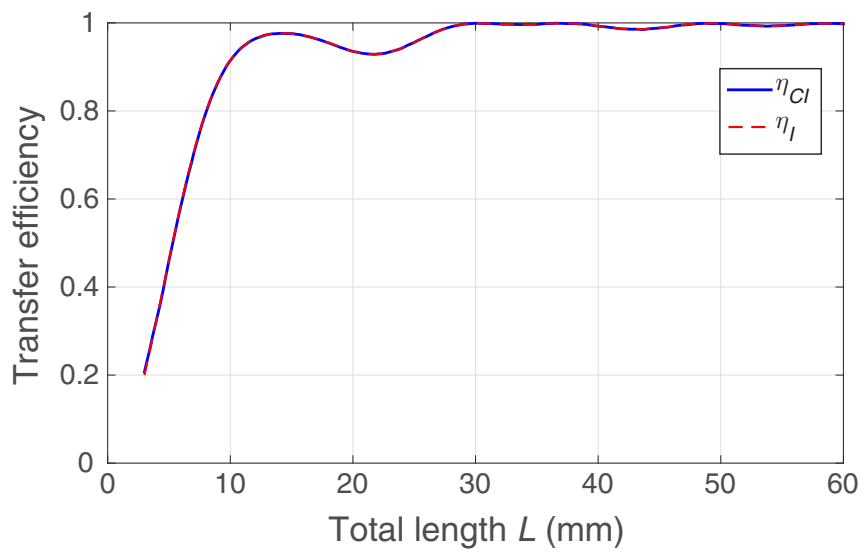

FIG. 7. Dependence of the transfer efficiencies $\eta_{C I}$ and $\eta_{I}$ on the total length $L$ of the structure for $\lambda=1.55 \mu \mathrm{m}$ and $N=3$. The geometrical functions $\delta x_{1}(z)$ and $\delta x_{N}(z)$ are given by the parabolas of Eqs. (19). The waveguide's shape still correspond to the one of Fig. 1(a) if $z$ is replaced by a normalized propagation distance $z / L$ and the same $x$ dimension in micrometers is kept.

of Fig. 7. The latter represents the variation of the transfer efficiency $\eta$ for the intuitive and counterintuitive cases as a function of $L$ and for $\lambda=1.55 \mu \mathrm{m}$. The curvatures of the two outer waveguides are still expressed by Eqs. (19). Therefore, a decrease (increase) of $L$ corresponds to a compression (expansion) of the structure of Fig. 1(a) in the $z$ direction, while keeping the $x$ dimensions unchanged. In this way, the distances between the waveguides at the entrance and output of the structure, as well as their minimum separations near $z=L / 2$, remain the same. Importantly, the central waveguide elimination criterion of Eq. (3) is not modified by a change of the total length $L$ since it is principally affected by the difference in refractive index contrast between the central and the outer waveguides. In contrast, the adiabatic condition of Eq. (12) depends on the geometrical shape and is decreasing by shortening $L$, thus the curves of Fig. 7 can be considered as a test of adiabaticity. It is clearly seen that $\eta_{C I}(L)$ and $\eta_{I}(L)$ overlap for all lengths $L$. This is related to the fact that the criterion (3) remains valid and therefore the coupler efficiency remains symmetric with only negligible transient light in waveguide 2, as in Figs. 2(c) and 2(d). The transfer efficiency in both directions remain high and exceeds $90 \%$ down to a length of about $10 \mathrm{~mm}$. Afterwards, the steep drop in efficiency seen for $L<10 \mathrm{~mm}$ is due to the loss of the adiabatic condition. Indeed, for $L=10 \mathrm{~mm}$, the adiabatic criterion (12) varies between 0.5 and 1.2 over the interacting (coupling) distance. For the chosen set of waveguide parameters, this limit value of $10 \mathrm{~mm}$ is consistent with the above discussion in connection to Fig. 3(b) and 3(c) since the crucial part of the $30-\mathrm{mm}$-long coupler is roughly $10-15 \mathrm{~mm}$. We verified also that the footprint of the three-waveguide coupler could be reduced as well by zooming on the central part of the coupler, effectively cutting away the initial and final parts of propagation with respect to Fig. 1(a). In this case, the waveguide curvatures are not changed. Also, in this situation, we have found a drop below $\eta<90 \%$ for $L<10 \mathrm{~mm}$. Note that in this case, for such a 10-mm-long coupler, the two outer waveguides are separated by $25 \mu \mathrm{m}$ (center to center) at 
$z=0$ or $z=L$ instead of the $\approx 55 \mu \mathrm{m}$ in Fig. 1(a). Note also that, even though the parameters of our original structure are optimized for a chosen length of $30 \mathrm{~mm}$, for shorter lengths (but $L \geqslant 10 \mathrm{~mm}$ ) the obtained efficiency may be further optimized by modifying (for each $L$ ) the curvature parameters in Eqs. (19) or by using a more complex geometrical function than the simple parabola considered in this proof-of-principle study.

\section{CONCLUSIONS}

In conclusion, we have theoretically proposed a method for light switching between the outer waveguides of a $\mathrm{N}$ waveguide array which provides the optical analog of the adiabatic elimination process used in quantum physics. Adiabatic elimination reduces the problem from a true $N$-waveguide system to an effective two-waveguide system with an effective pseudodetuning of the propagation constants. It was shown that, upon an appropriate choice of the longitudinal evolution of the coupling constants involving the outer waveguides, the above effective two-waveguide system behaves like a coupled two-level quantum system subjected to the rapid adiabatic passage process. In contrast to the case of the conventional
STIRAP process, for the present approach the photonic transport is found to be symmetric with respect to an interchange of the outer waveguides. The theoretical expectations have been successfully verified by means of wave propagation simulations by the beam propagation method applied to structures containing an odd $(N=3)$ or even $(N=6)$ number of waveguides. We have shown that for $N=3$ the system is very broadband and the transfer efficiency can be kept close to $100 \%$ over a wavelength range spanning more than $600 \mathrm{~nm}$ around the telecommunication wavelengths. For an increased number of waveguides $N$ in the structure, the bandwidth diminishes, but several wavelength regions with a nearly flat transfer efficiency close to $100 \%$ are still observed. Moreover, since the system behavior is influenced by higher-order modes in the central waveguide(s), slight modifications of the present technique might be useful also for novel approaches for mode conversion and filtering applications.

\section{ACKNOWLEDGMENTS}

This work has been supported by the France-Bulgaria bilateral program RILA and by the Region Lorraine.
[1] D. Dragoman and M. Dragoman, Quantum-Classical Analogies (Springer, Berlin, 2004).

[2] S. Longhi, Laser Photonics Rev. 3, 243 (2009).

[3] I. L. Garanovich, S. Longhi, A. A. Sukhorukov, and Yu. S. Kivshar, Phys. Rep. 518, 1 (2012).

[4] C. Rüter, K. G. Makris, R. El-Ganainy, D. N. Christodoulides, M. Segev, and D. Kip, Nat. Phys. 6, 192 (2010).

[5] A. A. Sukhorukov, Z. Xu, and Yu. S. Kivshar, Phys. Rev. A 82, 043818 (2010).

[6] M. C. Rechtsman, J. M. Zeuner, Y. Plotnik, Y. Lumer, D. Podolsky, F. Dreisow, S. Nolte, M. Segev, and A. Szameit, Nature (London) 496, 196 (2013).

[7] U. Gaubatz, P. Rudecki, S. Schiemann, and K. Bergmann, J. Chem. Phys. 92, 5363 (1990).

[8] K. Bergmann, H. Theuer, and B. W. Shore, Rev. Mod. Phys. 70, 1003 (1998).

[9] N. V. Vitanov, T. Halfmann, B. W. Shore, and K. Bergmann, Annu. Rev. Phys. Chem. 52, 763 (2001).

[10] N. V. Vitanov, M. Fleischhauer, B. W. Shore, and K. Bergmann, Adv. At. Mol. Opt. Phys. 46, 55 (2001).

[11] E. Paspalakis, Opt. Commun. 258, 31 (2006).

[12] S. Longhi, Phys. Rev. E 73, 026607 (2006).

[13] S. Longhi, G. DellaValle, M. Ornigotti, and P. Laporta, Phys. Rev. B 76, 201101(R) (2007).

[14] F. Dreisow, M. Ornigotti, A. Szameit, M. Heinrich, R. Keil, S. Nolte, A. Tünnermann, and S. Longhi, Appl. Phys. Lett. 95, 261102 (2009).

[15] G. Della Valle, M. Ornigotti, T. T. Fernandez, P. Laporta, S. Longhi, A. Coppa, and V. Foglietti, Appl. Phys. Lett. 92, 011106 (2008).

[16] A. A. Rangelov and N. V. Vitanov, Phys. Rev. A 85, 055803 (2012).

[17] C. Ciret, V. Coda, A. A. Rangelov, D. N. Neshev, and G. Montemezzani, Opt. Lett. 37, 3789 (2012).
[18] C. Ciret, V. Coda, A. A. Rangelov, D. N. Neshev, and G. Montemezzani, Phys. Rev. A 87, 013806 (2013).

[19] T. Y. Lin, F. C. Hsiao, Y. W. Jhang, C. Hu, and S. Y. Tseng, Opt. Express 20, 24085 (2012).

[20] S. Y. Tseng and X. Chen, Opt. Lett. 37, 5118 (2012).

[21] L. A. Lugiato, P. Mandel, and L. M. Narducci, Phys. Rev. A 29, 1438 (1984).

[22] A. Sinatra, F. Castelli, L. A. Lugiato, P. Grangier, and J. P. Poizat, Quantum Semiclass. Opt. 7, 405 (1995).

[23] M. P. Fewell, Opt. Commun. 253, 125 (2005).

[24] E. Brion, L. H. Pedersen, and K. Mølmer, J. Phys. A 40, 1033 (2007).

[25] B. W. Shore, Acta Phys. Slovaca 58, 243 (2008).

[26] S. Longhi, J. Phys. B 40, F189 (2007).

[27] M. Mrejen, H. Suchowski, T. Hatakeyama, Y. Wang, and X. Zhang, Nano Lett. 15, 7383 (2015).

[28] M. Mrejen, H. Suchowski, T. Hatakeyama, C. Wu, L. Feng, K. OBrien, Y. Wang, and X. Zhang, Nat. Commun. 6, 7565 (2015)

[29] T. Liu, A. S. Solntsev, A. Boes, T. Nguyen, C. Will, A. Mitchell, D. N. Neshev, and A. A. Sukhorukov, Opt. Lett. 41, 5278 (2016).

[30] H. Oukraou, L. Vittadello, V. Coda, C. Ciret, M. Alonzo, A. A. Rangelov, N. V. Vitanov, and G. Montemezzani, Phys. Rev. A 95, 023811 (2017).

[31] A. Yariv, IEEE J. Quantum Electron. 9, 919 (1973).

[32] C. Ciret, M. Alonzo, V. Coda, A. A. Rangelov, and G. Montemezzani, Phys. Rev. A 88, 013840 (2013).

[33] N. V. Vitanov, Phys. Rev. A 58, 2295 (1998).

[34] J. Van Roey, J. Van der Donk, and P. E. Lagasse, J. Opt. Soc. Am. 71, 803 (1981).

[35] K. Kawano and T. Kitoh, Introduction to Optical Waveguide Analysis Solving Maxwell's Equations and the Schrödinger Equation (Wiley, New York, 2001).

[36] I. H. Malitson, J. Opt. Soc. Am. 55, 1205 (1965). 